\title{
Acute vertigo in the emergency department: use of bedside oculomotor examination
}

Clovis J RAU ${ }^{1}$, Lovisa TERLING ${ }^{2}$, Samer ELKHODAIR ${ }^{1}$, Diego KASK| $\left.\right|^{3,4}$

Affiliations

1. Department of Emergency Medicine, University College London Hospitals, Gower Street, London, NW1 2BU, United Kingdom

2. Lund University, Box 117, SE-221 00, Lund, Sweden

3. Department of Clinical and Motor Neurosciences, 33 Queen Square, London WC1N 3BG

4. Department of Neuro-otology, Royal National ENT and Eastman Dental Hospitals, Huntley Street, London WC1E 6DG, United Kingdom

* Correspondence to: Dr Diego Kaski d.kaski@ucl.ac.uk; 00442033488782 
Vertigo is a sensation of false movement of the world, internal sensation of movement, tilt, or spatial disorientation. It is a common symptom reportedly experienced by nearly 1 in 4 adults in the United Kingdom (UK) as well as being one of the top reasons for patients to present to the Emergency Department (ED) [1]. Differentiating between peripheral (usually benign) and central (possibly sinister) causes of vertigo ranks among the highest challenges in Emergency Medicine [2], and the ability to do so relies upon experience in performing a focussed oculomotor examination [3].

The HINTS examination (an acronym of Head Impulse, direction-changing Nystagmus, and a Test of Skew) can be used to help identify posterior circulation strokes in patients with acute vertigo and one or more risk factors for stroke [4]. A positive HINTS examination involves a normal head impulse test, direction-changing nystagmus, and presence of a skew deviation. The combination of these three oculomotor findings has a sensitivity of $100 \%$ and specificity of $96 \%$ for stroke when performed by a specialist [5]. However, its use has not been validated in an ED setting, where it appears to be under-utilised [6]. Meanwhile, the Dix-Hallpike test is a diagnostic positional manoeuvre and is the only way to make a definitive diagnosis of benign paroxysmal positional vertigo (BPPV). Arguably, it should be performed in all patients presenting acutely with vertigo [7], given that BPPV is a common and treatable condition.

Here, we retrospectively evaluated current ED practice in the assessment, diagnosis, and management of 100 patients presenting with acute vertigo to a large University 
Hospital in the UK. Our primary aim was to establish the percentage of patients undergoing the Dix-Hallpike manoeuvre and HINTS examination in an ED setting. We conducted a retrospective case note review of adult patients presenting to the Emergency Department at University College London Hospital with a chief complaint of 'dizziness', 'vertigo', 'gait problem' or 'gait disturbance'. 100 sequential cases with symptoms indicative of vertigo were included. Using a proforma, we collected information on the nature and duration of symptoms, past medical history, medications and vascular risk factors. We recorded the initial examination findings, including the use of the HINTS examination and Dix-Hallpike manoeuvre, and diagnosis both in ED and at any outpatient follow-up performed in the 6 weeks following initial attendance. Data was collected by two independent practitioners using the hospital's electronic health record (Epic, Epic Systems Corporation).

Of the 100 patients included in the study, 43 were male and 57 were female (male:female ratio 1:1.33). The mean age at presentation was 49.8 years. The HINTS examination was performed on 18 patients. The Dix-Hallpike manoeuvre was performed on 13 patients, and an Epley manoeuvre performed on 7 patients (Figure 1A). Only 5 patients underwent both a HINTS examination and Dix-Hallpike manoeuvre, and no patients had a bedside test of hearing documented. Nystagmus was present in 25 patients, absent in 63 , and not documented in 12 . The most common final diagnosis, as determined by the discharging team, was BPPV $(43 \%)$, followed by acute vestibular neuritis (26\%), migraine (6\%), postural/orthostatic hypotension (4\%), ear infections (3\%), Ménière's disease (2\%) and stroke (2\%). Eighteen patients were admitted, 12 under medicine, 6 under stroke. 
In a short time following the original description, the HINTS examination has become increasingly popular among neurologists in the evaluation of patients with acute vertigo. Data suggests that its use may reduce rates of misdiagnosis, with associated reduction of disease burden for the individual and healthcare. Despite this, we found a low rate $(18 \%)$ of application in the ED. We suspect this is due to clinician unfamiliarity with the test, lack of training in eye movement assessment, and low confidence with its interpretation.

Nystagmus plane (horizontal, vertical, torsional), direction (left-beating, rightbeating), and change with gaze were scarcely documented, in keeping with other studies [8]. Whilst there was a tendency to focus on the direction of gaze where nystagmus was seen (e.g. 'nystagmus on leftward gaze') there was insufficient detail to interpret the nystagmus plane, direction, and whether it changed with gaze - a key requirement for the HINTS examination.

Of the patients seen and discharged by ED physicians, 41 were given a diagnosis of BPPV. However, of these, only 10 (24\%) had undergone a Dix-Hallpike manoeuvre to elicit the diagnosis [9]. Although there is no evidence base for medication in the management of BPPV [7], 21 of the 41 patients who were given an ED diagnosis of BPPV were discharged with a course of prochlorperazine, rather than correcting the underlying pathology with a repositioning manoeuvre [10]. 
The low rates of Dix-Hallpike manoeuvres performed renders evaluation of the true incidence of BPPV difficult. We suspect that a proportion of patients with an ED diagnosis of BPPV may in fact have other vestibular causes for their symptoms, most notably vestibular migraine [11], a common diagnosis that was not recorded for any patient in our cohort, and which tends to occur in younger populations [12].

In the 26 patients where specialty teams were involved with the patient's care as either an inpatient or outpatient, the initial ED diagnosis was revised in 12 cases (46\%, Figure 1B), suggesting that acute ED diagnosis of dizziness and vertigo is inaccurate. Specialty teams included acute medicine, stroke, and ear, nose and throat (ENT), and revisions to diagnosis were based on further imaging, re-eliciting the history, serial clinical examination and outpatient follow-up.

Our study suggests that UK ED clinicians are not confident in using clinical examination tools such as the HINTS examination and the Dix-Hallpike manoeuvre to distinguish between peripheral and central causes of vertigo. There is a need for educational programmes to enhance the use of bedside examination in the assessment of the acutely dizzy patient, and prospective studies with formal neurootological follow-up to elicit the true incidence of conditions presenting with acute vertigo to UK EDs. 
Acknowledgements

Conflicts of Interest

There are no conflicts of interest. 


\section{Captions}

Figure 1. A: Euler diagram illustrating the relationship between patients with an ED diagnosis of BPPV, the use of Dix-Hallpike manoeuvre, and the administration of phenothiazines. B. Rate and type of misdiagnosis where a specialty team was involved, either in outpatient or inpatient care, following initial diagnosis by the emergency department. $A V N=$ acute vestibular neuritis; $B P P V=$ benign paroxysmal positional vertigo; $\mathrm{OH}=$ orthostatic hypotension. 


\section{References}

1 Murdin L, Schilder AGM. Epidemiology of Balance Symptoms and Disorders in the Community. Otol Neurotol 2015; 36:387-92.

2 Eagles D, Stiell IG, Clement CM, Brehaut J, Kelly AM, Mason S, et al. International survey of emergency physicians' priorities for clinical decision rules. Acad Emerg Med 2008; 15:177-82.

3 Edlow JA, Newman-Toker D. Using the Physical Examination to Diagnose Patients with Acute Dizziness and Vertigo. J Emerg Med 2016; 50:617-28.

4 Cohn B. Can Bedside Oculomotor (HINTS) Testing Differentiate Central From Peripheral Causes of Vertigo? Ann Emerg Med 2014; 64(3):265-8.

5 Newman-Toker DE, Kerber KA, Hsieh YH, Pula JH, Omron R, Saber Tehrani AS, et al. HINTS outperforms ABCD2 to screen for stroke in acute continuous vertigo and dizziness. Acad Emerg Med 2013; 20:987-96.

6 Kohn MA. HINTS to identify stroke in ED patients with dizziness. Acad Emerg Med 2014;21(3):347.

$7 \quad$ Kaski D, Agarwal K, Murdin L. Acute Vertigo. BMJ 2019; 366:I5215.

8 Kerber KA, Morgenstern LB, Meurer WJ, McLaughlin T, Hall PA, Forman J, et al. Nystagmus assessments documented by emergency physicians in acute dizziness presentations: A target for decision support? Acad Emerg Med 2011; 18:619-26.

9 Bhattacharyya N, Gubbels SP, Schwartz SR, Edlow JA, El-Kashian H, Fife T, 
et al. Clinical Practice Guideline: Benign Paroxysmal Positional Vertigo (Update). Otolaryngol Neck Surg 2017; 156:S1-47.

10 Kaski D, Bronstein AM. Epley and beyond: an update on treating positional vertigo. Pract Neurol 2014; 14:1-12.

11 Kheradmand A, Bronstein A, Zee DS. Clinical Bedside Examination. In: Oxford Textbook of Vertigo and Imbalance. 2013.

12 Dieterich M, Obermann M, Celebisoy N. Vestibular migraine: the most frequent entity of episodic vertigo. J Neurol 2005. 263:82-89. 